\title{
Estimativa do desperdício das dietas hospitalares associado a satisfação e aceitação dos pacientes no Hospital Universitário Alcides Carneiro em Campina Grande -PB
}

Estimatimate of waste of hospital diets associated with patient satisfaction and acceptance at the University Hospital Alcides Carneiro in Campina Grande -PB

Estimación de residuos de dietas hospitalarias asociado a la satisfacción y aceptación del paciente en el Hospital Universitario Alcides Carneiro de Campina Grande -PB

Recebido: 12/01/2021 | Revisado: 14/01/2021 | Aceito: 15/01/2021 | Publicado: 18/01/2021

Sandra Regina Dantas Baía ORCID: https://orcid.org/0000-0002-0777-176X Universidade Federal de Campina Grande, Brasil E-mail: sandra_reginabaia@hotmail.com

Thaisa Abrantes da Silva Souza ORCID: https://orcid.org/0000-0001-8640-7036 E-mail: ta_brantes@hotmail.com Universidade Federal de Campina Grande, Brasil

Rennan Pereira de Gusmão ORCID: https://orcid.org/0000-0002-7355-8078 Universidade Federal de Campina Grande, Brasil E-mail: rennangusmao@gmail.com

Rebeca Morais Silva Santos ORCID: https://orcid.org/0000-0003-0867-2795 Universidade Federal de Campina Grande, Brasil E-mail: rebecamoraiscg@gmail.com

Gabriela Mariana Cruz Pimentel ORCID: https://orcid.org/0000-0003-3924-833X Centro Universitário Maurício de Nassau, Brasil E-mail: gabipimentel.nutri@gmail.com

Rikaelly Vital Costa

ORCID: https://orcid.org/0000-0002-2570-7083 Centro Universitário Maurício de Nassau, Brasil E-mail: rikaellyv@gmail.com

Elis Dantas Medeiros

ORCID: https://orcid.org/0000-0003-2328-6497 Universidade Federal da Paraíba, Brasil E-mail: elisdantas.arq@gmail.com

\section{Resumo}

Vários são os impactos causados ao meio ambiente ocasionados pelos processos de produção de refeições. A pesquisa foi realizada com o objetivo de avaliar o desperdício dos alimentos associados a aceitação e a satisfação da dieta hospitalar dos pacientes internos do Hospital Universitário em Campina Grande-PB. Trata-se de um estudo transversal quantitativo, onde foi aplicado o sistema de pesagem antes e após a refeição e posteriormente foi aplicado um questionário referente a satisfação das dietas oferecidas aos internos. Destacamos a opção de muito satisfeitos para os atributos: sabor 33,98\%; aparência 33\%; cor 35,92\%; textura 33,98\%; temperatura 46,60\%; forma de distribuição $51,45 \%$; recipiente servido $53,39 \%$ e quantidade servida $41,74 \%$. Embora o estudo apresente uma boa satisfação na dieta ofertada, ainda assim há um desperdício com um valor de $30 \%$, o que ultrapassa o limite aceitável de $20 \%$ para coletividade enferma, demonstrando um consumo reduzido de ingestão de energia e proteína pelo paciente, ocasionando um duplo aumento no risco de morte durante uma internação hospitalar. Em relação aos dois gêneros constatamos que o gênero masculino apresentou um menor desperdício $(27,88 \%)$ em relação ao gênero feminino $(32,79 \%)$. Corroborar a importância de ações educativas com intuito de amenizar esses desperdícios alimentares e evitar desnutrição nos pacientes, custos hospitalares e impactos ao meio ambiente, realizando trimestralmente uma pesquisa de satisfação com os pacientes; expor em murais a quantidade de desperdício diária dos alimentos; e doar os alimentos excedentes a pessoas com risco alimentar e nutricional, apoiado na Lei 14016 de 23 de junho de 2020.

Palavras-chave: Impacto ambiental; Dieta; Perdas; Hospital.

\section{Abstract}

There are several impacts to the environment caused by meal production processes. The research was carried out with the objective of evaluating food waste associated with acceptance and satisfaction of the hospital diet of inpatients at 
the University Hospital in Campina Grande-PB. This is a quantitative cross-sectional study, where the weighing system was applied before and after the meal and subsequently a questionnaire was applied regarding the satisfaction of the diets offered to the interns. We highlight the option of very satisfied for the attributes: flavor 33.98\%; appearance 33\%; color $35.92 \%$; texture $33.98 \%$; temperature $46.60 \%$; distribution method $51.45 \%$; container served $53.39 \%$ and quantity served $41.74 \%$. Although the study shows good satisfaction in the offered diet, there is still a waste with a value of $30 \%$, which exceeds the acceptable limit of $20 \%$ for the sick community, demonstrating a reduced consumption of energy and protein intake by the patient, causing a double increase in the risk of death during a hospital stay. Regarding both genders, we found that the male gender had a lower waste $(27.88 \%)$ compared to the female gender $(32.79 \%)$. Corroborate the importance of educational actions in order to mitigate these food waste and avoid malnutrition in patients, hospital costs and impacts on the environment, conducting a patient satisfaction survey every three months; display on murals the amount of daily food waste; and donating surplus food to people at food and nutritional risk, supported by Law 14016 of 23 June 2020.

Keywords: Environmental impact; Diet; Losses; Hospital.

\section{Resumen}

Existen varios impactos en el medio ambiente causados por los procesos de producción de harinas. La investigación se realizó con el objetivo de evaluar el desperdicio de alimentos asociado a la aceptación y satisfacción de la dieta hospitalaria de los pacientes hospitalizados del Hospital Universitario de Campina Grande-PB. Se trata de un estudio cuantitativo transversal, donde se aplicó el sistema de pesaje antes y después de la comida y posteriormente se aplicó un cuestionario sobre la satisfacción de las dietas ofrecidas a los pasantes. Destacamos la opción de muy satisfecho por los atributos: sabor 33,98\%; apariencia 33\%; color 35,92\%; textura 33,98\%; temperatura 46,60\%; método de distribución 51,45\%; envase servido 53,39\% y cantidad servida $41,74 \%$. Si bien el estudio muestra una buena satisfacción en la dieta ofrecida, aún existe un desperdicio con un valor del $30 \%$, que supera el límite aceptable del $20 \%$ para la comunidad enferma, demostrando un menor consumo de energía y aporte proteico por parte del paciente, provocando un doble aumento del riesgo de muerte durante la estancia hospitalaria. Respecto a ambos géneros, encontramos que el género masculino presentó un menor desperdicio $(27,88 \%)$ en comparación con el género femenino (32,79\%). Corroborar la importancia de las acciones educativas para mitigar estos desperdicios de alimentos y evitar la desnutrición en los pacientes, los costos hospitalarios y los impactos en el medio ambiente, realizando una encuesta de satisfacción del paciente cada tres meses; mostrar en murales la cantidad de desperdicio diario de alimentos; y donación de excedentes de alimentos a personas en riesgo alimentario y nutricional, amparado por la Ley 14016 de 23 de junio de 2020.

Palabras-clave: Impacto medioambiental; Dieta; Pérdidas; Hospital.

\section{Introdução}

Vários são os impactos causados ao meio ambiente ocasionados pelos processos de produção de refeições, pois são consumidos materiais, energia e água, utilizando equipamentos, recursos humanos e instalações físicas e produzem resíduos sólidos, efluentes e emissões de gases para a atmosfera, além do seu produto final, a refeição (Strasburg \& Jahno, 2017).

A unidade de alimentação e nutrição (UAN) é a unidade de trabalho que desempenha atividades técnicoadministrativas necessárias à manipulação, à preparação, ao armazenamento e à distribuição de alimentos e de refeições nutricionalmente balanceadas e precisam apresentar-se seguras microbiologicamente, assim como fornecer alimentos adequados às necessidades específicas do cliente/paciente, tanto para as coletividades sadias quanto para as enfermas. (Wendisch, 2010).

Os hospitais desempenham um importante papel na alimentação, devido ao seu grande poder de compra, uso de recursos e geração de resíduos. Os sistemas alimentares hospitalares incluem serviço de alimentação para nutrição dos pacientes, bem como serviço de alimentação para funcionários e acompanhantes. Dependendo de sua condição, alguns pacientes podem receber, além de refeições, suplementos de nutrição oral e alimentos entéricos ou parenterais exclusivamente ou em combinação. E por esse fato, os serviços de alimentação hospitalares podem afetar negativamente o ambiente em quaisquer fases da cadeia de abastecimento alimentar (produção / aquisição, distribuição, preparação gestão, consumo e gestão / eliminação de resíduos) (Carino, Porter, Malekpour, \& Collins, 2020).

Em vários hospitais são constatados desperdícios de alimentos que podem chegar até 30\%, sendo esse valor acima da média de outros serviços de alimentação. E, estando associado à redução da ingestão de energia e proteína ocasionando um 
duplo aumento no risco de um paciente morrer durante uma internação hospitalar se comem apenas um quarto da comida fornecida. Lamentavelmente há poucas de evidências para apoiar a intervenção soluções para melhorar este problema (Williams \& Walton, 2011).

De acordo com Duarte, Marques, \& Colpo (2016), a desnutrição hospitalar ocorre em cerca de $20 \%$ a $50 \%$ dos pacientes. Porém, alguns destes já surgem ao hospital com desnutrição, e outros a adquirem durante sua permanência, influenciando assim o agravamento do seu estado de saúde, uma vez que há uma queda na atividade do sistema imunológico pela deficiência de nutrientes necessários para uma defesa imunológica.

O fato de alguns pacientes já chegarem aos hospitais com sinais e sintomas de desnutrição pode estar associado às causas do seu estilo de vida, má alimentação, patologias associadas, condições socioeconômicas, alterações psíquicas, condições fisiológicas, como por exemplo, perda da dentição e consequentemente problemas na mastigação, disfagia, poli farmácia etc (Silva, Marques, Leal, Alencar, \& Melo, 2015).Além do estado nutricional pré-hospitalar, existem fatores importantes que interferem no estado nutricional do indivíduo durante sua estadia ao hospital, dentre elas, a aceitação e satisfação da dieta hospitalar (Soares, 2015).

Desta forma, o presente estudo teve objetivo avaliar o desperdício dos alimentos associados à satisfação e à aceitação da dieta hospitalar dos pacientes internos do Hospital Universitário Alcides Carneiro na cidade de Campina Grande-PB.

\section{Metodologia}

O presente estudo transversal de cunho quantitativo foi realizado no Hospital Universitário Alcides Carneiro localizado na cidade de Campina Grande-PB, durante um período de 10 dias, alternados entre os meses de setembro a dezembro de 2020. O método pra ser quantitativo deve adquirir dados quantitativos e numéricos e posteriormente analisá-los por meio de técnicas matemáticas (porcentagens, estatísticas e probabilidades, métodos numéricos, métodos analíticos e geração de equações e/ou fórmulas matemáticas. (Pereira et al., 2018)

A pesquisa foi aprovada pelo Comitê de Ética (CEP/HUAC) gerando o CAAE:33931920.1.0000.5182, respeitando todas as recomendações da Resolução ${ }^{\circ}$ 466/2012. Para não expor a identidade dos voluntários foi desenvolvido um código para identificá-los de forma sigilosa.

A pesquisa de campo foi realizada com 103 pacientes internos. Estes foram selecionados através de dados coletados nos prontuários eletrônicos. Os critérios de inclusão foram: pacientes adultos com faixa etária a partir de 18 anos de idade, internos apenas nas áreas clínicas e com condições físicas e psicológicas preservadas. Os critérios de exclusão foram: pacientes impossibilitados de se comunicar e que não estejam alimentando-se por via oral. Posteriormente foram esclarecidos sobre a pesquisa e submetidos a aplicação prévia do TCLE (Termo de consentimento livre e esclarecido).

Inicialmente foi realizada a pesagem apenas da refeição do almoço oferecida aos pacientes ainda na área de produção da UAN, considerando a prescrição das dietas realizadas pelas Nutricionistas do hospital. O peso foi medido em gramas utilizando uma balança digital de cozinha, SF-400, com capacidade de $10 \mathrm{~kg}$ e precisão de $0,1 \mathrm{~g}$. Após a distribuição da refeição, as sobras eram pesadas pelas pesquisadoras, retirando ossos e aparas e o peso da embalagem de isopor, que podem influenciar na possível verificação do desperdício.

Para fins de cálculos foram utilizados os indicadores de resto-ingesta, conforme Abreu (2016) adaptado, segundo as equações 1,23 e 4 : 


\begin{tabular}{|c|c|}
\hline 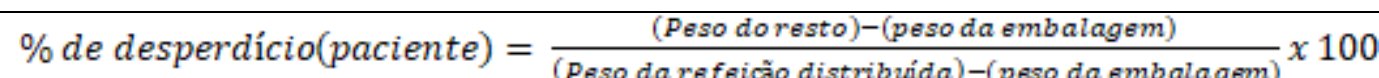 & (Eq. 1) \\
\hline$\%$ de consumo $($ paciente $)=100 \%-\%$ de desperdício $($ pacente $)$ & (Eq. 2) \\
\hline$\%$ de desperdício $($ total $)=\frac{\Sigma(\text { peso doresto })-\Sigma(\text { peso das embalagens })}{\Sigma(\text { Peso da ref eiçẩo distribuída })-\Sigma(\text { peso da embalagem) }} \times 100$ & (Eq. 3) \\
\hline$\%$ de consumo $($ total $)=100 \%-\%$ de desperdício $($ total $)$ & (Eq. 4) \\
\hline
\end{tabular}

Em seguida foi aplicado um questionário de aceitação alimentar "Pesquisa de satisfação de dieta hospitalar" elaborado e adaptado pela pesquisadora baseado no questionário de aceitabilidade de Coloço, Holanda e Portero (2009).

No questionário foram avaliados os atributos das refeições como: sabor, aparência, cor, textura, temperatura, forma de distribuição, recipiente servido e quantidade servida. O grau de satisfação foi mapeado utilizando os indicadores de: "Muito satisfeito", "Satisfeito", "Pouco satisfeito" e "Insatisfeito". Também foram questionados sobre o seu estado de saúde naquele dia, bem como o seu apetite antes d.

Após a aplicação dos instrumentos de coleta de dados, foi fornecido um copo de material acrílico com o intuito de incentivar os participantes a utilizarem-no nas dependências do hospital, sendo um passo inicial para uma futura educação ambiental, evitando o uso de descartáveis.

Os dados foram organizados no Microsoft Office Excel (2019) e gerados gráficos e equações para interpretação dos resultados obtidos.

\section{Resultados e Discussão}

O presente estudo incluiu 103 pacientes com a mediana de idade de 58,81 anos. Do total de pacientes, 53,39\%(n=55) possuíam idade superior a 60 anos, classificados como idosos. Em relação ao gênero foram classificados 45,63\% do gênero feminino (n=47) e 54,36\% ( $\mathrm{n}=56$ ) do gênero masculino, como demonstra a Figura 1.

Figura 1. Perfil dos pacientes participantes da pesquisa.

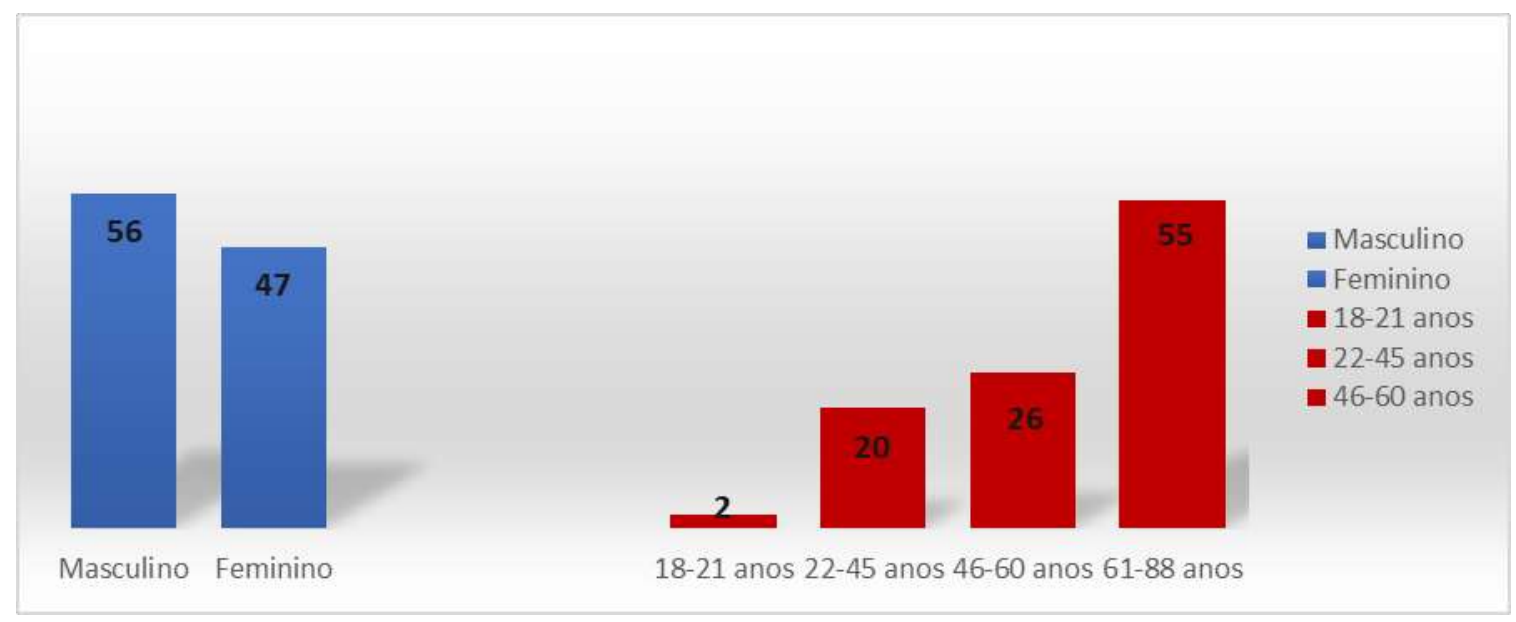

Fonte: Autores (2020). 
No Quadro 1, são apresentadas as comorbidades dos pacientes, onde constata-se a presença de patologias diversas especialidades, com maior destaque as doenças endócrinos/metabólicas e neoplasia diversas, onde há uma certa restrição alimentar, que deve ser avaliada pelo estado geral do paciente.

Quadro 1. Patologias dos pacientes participantes da pesquisa.

\begin{tabular}{|c|c|c|}
\hline Comorbidades & Patologias & Quantidades de pacientes \\
\hline $\begin{array}{l}\text { Doenças do sistema endócrino e } \\
\text { metabólicas }\end{array}$ & $\begin{array}{lll}\text { Diabetes mellitus; } & \text { transtorno } \\
\text { suprarrenal } & & \\
\end{array}$ & 23 \\
\hline Distúrbios hidroeletrolíticos & Hipoosmolaridade e hiponatremia & 1 \\
\hline Doenças cerebrovasculares & AVC & 1 \\
\hline Doenças pulmonares & $\begin{array}{l}\text { DPOC; Asma; } \quad \text { Pneumonia; } \\
\text { pulmonar primária; derrame pleural }\end{array}$ & 6 \\
\hline Neoplasias & $\begin{array}{l}\text { cabeça; tireoide; estômago; } \\
\text { duodeno; bexiga; coluna; pênis; } \\
\text { peritônio; massa tumoral }\end{array}$ & 20 \\
\hline Doenças hepáticas/baço: & $\begin{array}{l}\text { Abscesso hepático; esplenomegalia; } \\
\text { cirrose hepática; hepatite alcóolica; } \\
\text { Fibrose esclerose alcoólica do } \\
\text { fígado; }\end{array}$ & 11 \\
\hline Doenças cardiovasculares & Aterosclerose; endocardite; ICC & 6 \\
\hline Doenças hematológicas & $\begin{array}{l}\text { Anemias; Defeito qualitativo das } \\
\text { plaquetas; órgãos } \\
\text { hematopoiéticos;trobocistopenia; } \\
\text { leucemia mieloide crônica }\end{array}$ & 10 \\
\hline Virose & Dengue & 1 \\
\hline Doenças de pele/imunológico & $\begin{array}{l}\text { Erisipela; furúnculo; pelagra; } \\
\text { Pênfigo bolhoso; psoríase; }\end{array}$ & 7 \\
\hline Doença reumatológica & Lúpus eritematoso sistêmico (LES) & 6 \\
\hline Doença do trato digestivo & Esofagite tuberculosa; úlceras & 4 \\
\hline Doença hereditária & Fibrose cística & 1 \\
\hline Doenças infecciosas & $\begin{array}{l}\text { Bacteriana; trato urinário; óssea; } \\
\text { Pielonefrite }\end{array}$ & 5 \\
\hline Doença renal & Síndrome nefrítica aguda; & 1 \\
\hline
\end{tabular}

Fonte: Autores (2020).

Na Figura 2 pode ser observada a análise do paciente em relação ao seu estado de saúde no dia da pesquisa. 
Figura 2. Análise dos pacientes em relação à sua saúde no dia da coleta dos dados.

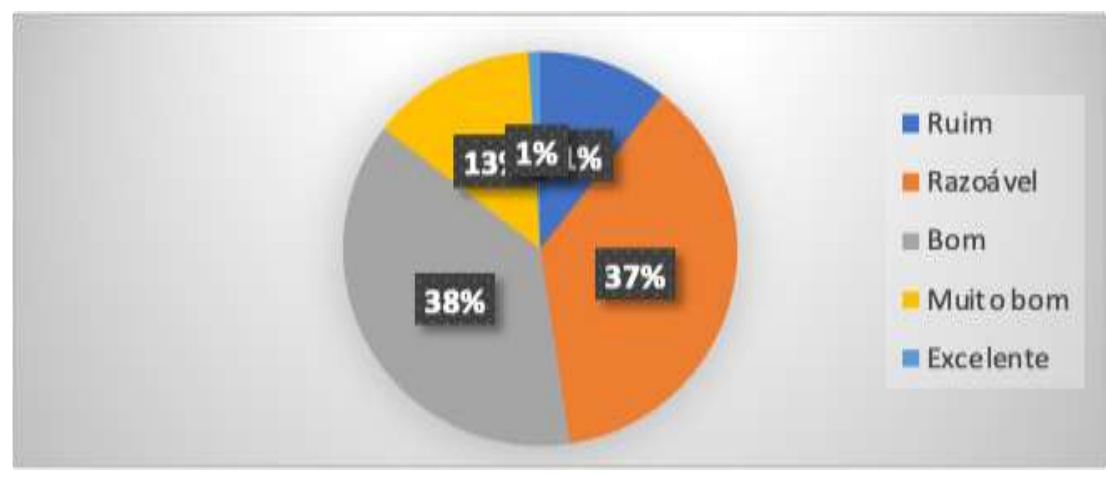

Fonte: Autores (2020).

Evidencia-se que a autoanálise do paciente se dá de forma contraditória, visto que, 38\%(n=39) declara está se sentindo em um bom estado de saúde e $37 \%(n=38)$ em estado razoável em relação a sua saúde. Comparando com o estudo de Wolff, Flynn, Xuan, Errichetti, Walker e Brodesky (2020), que analisaram a relação entre a saúde física e mental dos pacientes diabéticos e concluiu que existe uma necessidade permanente de melhorar a atenção à saúde desses pacientes, com a implantação de cuidados mais integrados nos serviços de saúde e o quanto é importante essa autoanálise para a melhoria do quadro do paciente.

Observa-se na Figura 3 as respostas dos pacientes ao questionamento sobre seu estado de fome, antes de consumir a refeição.

Figura 3. Análise do paciente em relação ao seu estado de fome no ato da pesquisa.

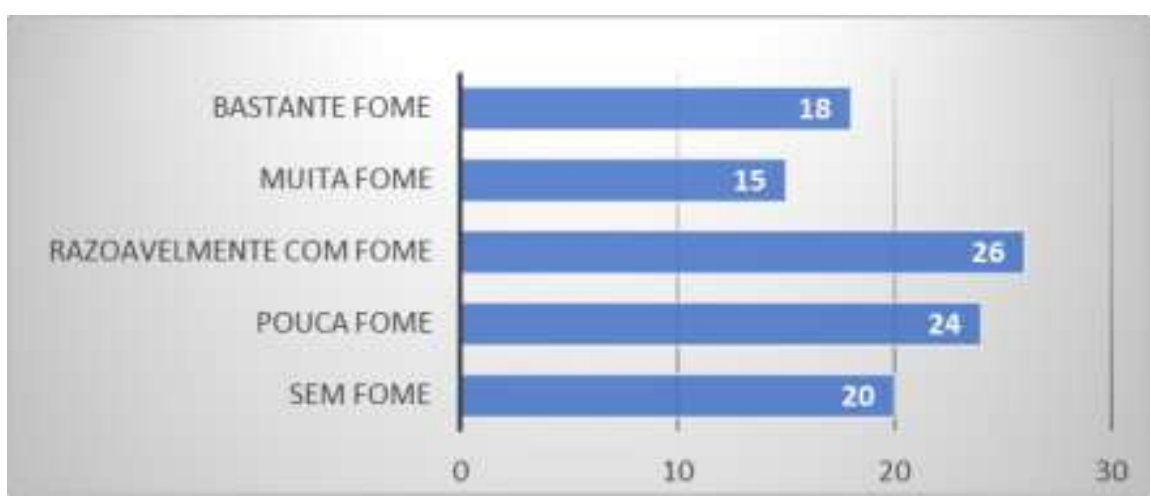

Fonte: Autores (2020).

Podemos observar que $17,47 \%(n=18)$ apresentava-se com bastante fome na horada refeição; $14,5 \%(n=15)$ com muita fome; 25,24 $n=26)$ com fome razoável; 23,30\%(n=24) relata que estavam com pouca fome e 19,41\%(n=20) que estavam sem fome antes da refeição. Como a maioria dos pacientes são idosos, essa diminuição do apetite pode ser devida as distorções das funções gustativas que ocorrem nessa fase da vida e que são agravadas por certas condições médicas, intervenções farmacológicas, radiação e exposição a produtos químicos tóxicos (Schiffman, 2009).

Na Figura 4 são apresentados os tipos de dietas oferecidas a esses pacientes, baseada nas suas patologias e que são prescritas diariamente pela equipe médica e nutricional, mediante também visitas ao leito do paciente para verificação de possíveis mudanças e evolução na conduta. Foi observado que a média diária de permanência desses pacientes foi de 8,4 dias, dando destaques a alguns pacientes que estavam há 47 dias internados. A pesquisa teve um cuidado de excluir entrevistados 
que passaram mais de dois meses internados no hospital, pois esse fator provavelmente iria interferir no resultado, devido as repetições de preparações.

Figura 4. Tipos de dietas hospitalares prescritas.

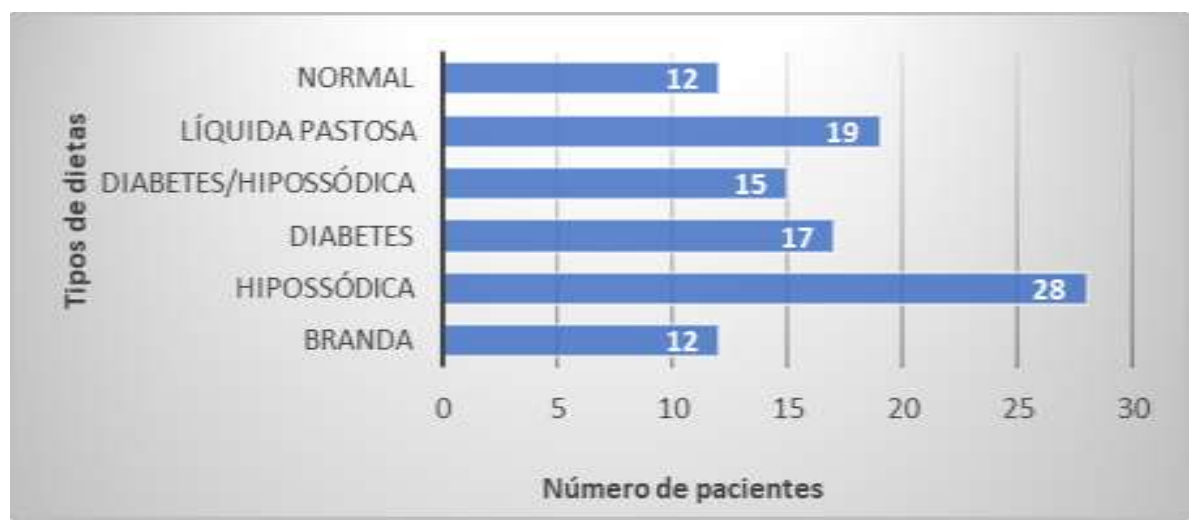

Fonte: Autores (2020).

Quando foram analisadas as dietas ofertadas destacamos três mais frequentes: 18,44\% (n=19) dos pacientes estavam consumindo uma dieta líquida pastosa, essa por sua vez é prescrita para pacientes com dificuldade de deglutição e mastigação. E, segundo Mancopes, Gonçalves, Costa, Flores, Santos e Drozdz (2013) a deglutição é influenciada pelas ressecções laríngeas, resultando em disfagia mecânica, caracterizada pela dificuldade em engolir na etapa orofaríngea, o controle neurológico central e os nervos periféricos permanecem intactos e tudo isso pode afetar a aceitação da dieta pastosa. Além disso, a disfagia pode estragar a progressão alimentar através da trato digestivo resultando na entrada do alimento nas vias aéreas, dando origem a tosse, sufocação / asfixia, aspiração, problemas pulmonares e, broncopneumonia por aspiração que é a complicação mais grave.

Em seguida o maior número apresentado foi das dietas hipossódicas, perfazendo um percentual de 27,18\%(n=28) dos internos. Quando comparamos com o estudo de Macedo, Pereira, Oliveira e Magalhães (2017), este apresenta que a dieta hipossódica é uma das dietas hospitalares que possui menor aceitação, devido a questão cultural que esse mineral exerce sobre a população em geral, estando presente em quantidades relevantes nas refeições domiciliares e quando apenas reduzido na dieta hospitalar isso gera uma baixa aceitação e desperdício alimentar.

Em um maior número 31,06\% $(\mathrm{n}=32)$ são apresentadas as dietas para diabéticos e em 15 destes pacientes encontramos a restrição do sal, dificultando mais ainda a aceitação da dieta. Um agravante é que a maioria dos pacientes diabéticos realizam atividades inadequadas de autocuidado, com pouco conhecimento do real controle sobre sua doença e isso influencia na adesão a uma boa alimentação ao logo do seu tratamento (Vilchez-Cornejo, Romani, Reategui, Gomez-Rojas, \& Silva, 2020).

Na Figura 5 pode ser encontrada o grau de satisfação dos pacientes em relação a sua satisfação da dieta ofertada pelo hospital durante o período de internação. 
Figura 5. Satisfação das dietas avaliadas pelos pacientes durante a coleta de dados.

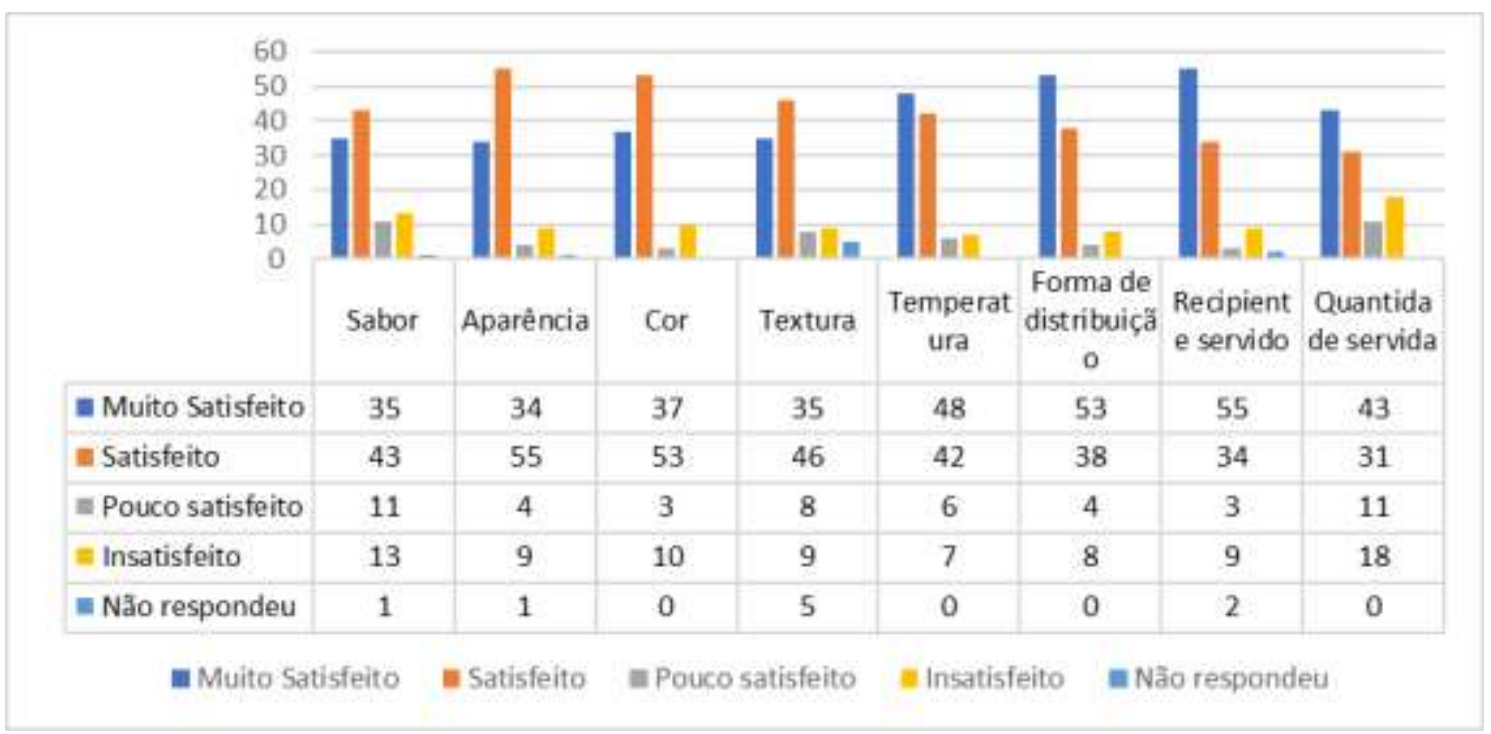

Fonte: Autores (2020).

Todos os critérios analisados obtiveram destaque para o item de muito satisfeito, isto é, as refeições servidas foram bem aceitas pelos pacientes. Os percentuais de aprovação para o item muito satisfeito foram de 33,98\%(n=35) para o item sabor; 33\% ( $\mathrm{n}=34)$ para a aparência, demonstrando que a apresentação era visualmente agradável, bem como a cor, que apresentou o resultado de 35,92\%(n=37); a textura por sua vez obteve 33,98\% (n=35) de muita satisfação, onde foram avaliadas as características de qualidade intrínsecas e extrínsecas dos alimentos; e a temperatura atingiu 46,60\%(n=48) de muita satisfação. Em relação ao recipiente servido, é utilizada uma embalagem de isopor (com capacidade média de 750 g) e que são preparadas ainda na área de produção com a identificação do paciente e o tipo de dieta e quando foi questionado sobre esse recipiente, $53,39 \%(\mathrm{n}=55)$ dos internos o classificaram como muito satisfeito. E por fim, quando questionadas sobre a quantidade servida, esta apresentou muita satisfação com alcance de 41,74\%(n=43).

Esse grau de satisfação por parte dos pacientes em relação a refeição servida no HUAC corrobora com o estudo de Mosqueira e Harris (1996) e Folio, Osullivan-Maillet e Touger-Decker (2002), quando destacam que a atividade do Nutricionista de realizar visitas ao leito e disponibilizar a escolha dos cardápios de acordo com a situação de saúde do paciente e disponibilidade de produtos no estabelecimento de saúde, melhorou a satisfação dos pacientes em relação às suas refeições (Vaillant, Alligier, Baclet, Capelle, Dousseaux, Eyraud, Fayemendy, Flori, Guex; \& Hennequin, 2019).

De acordo com Nascimento, Moreira, Carvalho, Pereira, Pereira e Vilela (2017), que realizaram um estudo sobre aceitabilidade das dietas hospitalares em um Hospital de Minas Gerais, e que foram aplicados 26 questionários em pacientes, com objetivo de identificar a aceitação dos pacientes em relação à refeição ofertada. A alimentação foi considerada boa ou ótima por $88,5 \%$ dos pacientes, onde foram analisadas características como: sabor, aparência, temperatura, variedade, higiene, utensílios, forma de distribuição, textura, horários e cortesia. Nesse estudo, o indicador higiene teve destaque com um percentual 30,8\% considerado muito importante e indicadores sem muita importância foram destacados como os utensílios e a forma de distribuição com $19,2 \%$ e $3,9 \%$, respectivamente.

Destacamos, em nosso estudo, que $17,47 \%(n=18)$ dos pacientes relataram que estavam insatisfeitos com a porção servida pois ultrapassava suas necessidades e com isso gerava desperdícios. 
Um estudo realizado na Itália mostrou que cerca de $41,6 \%$ da comida servida a pacientes internados em três hospitais era desperdiçada e que além de gerar muitos impactos ambientais, foi comprovado que a maioria dos hospitais não possuem o conhecimento deste fato e que isso gera muitos custos adicionais. As medidas preventivas sugeridas aos hospitais para combater desperdício dos alimentos (FW) foram estabelecer um serviço de alimentação de refeição individualizada, para simplificar e flexibilizar o processo de produção de refeição com base em necessidades nutricionais específicas, preferências e escolhas do paciente e para melhorar o serviço de alimentação baseado em pesquisas de satisfação do consumidor (Gomes, Saraiva, Esteves, \& Gonçalves ,2020).

A Figura 6 representa a análise do total de alimentos consumidos e os desperdiçados no período da coleta.

Figura 6. Consumo versus desperdício em relação ao total de produção.

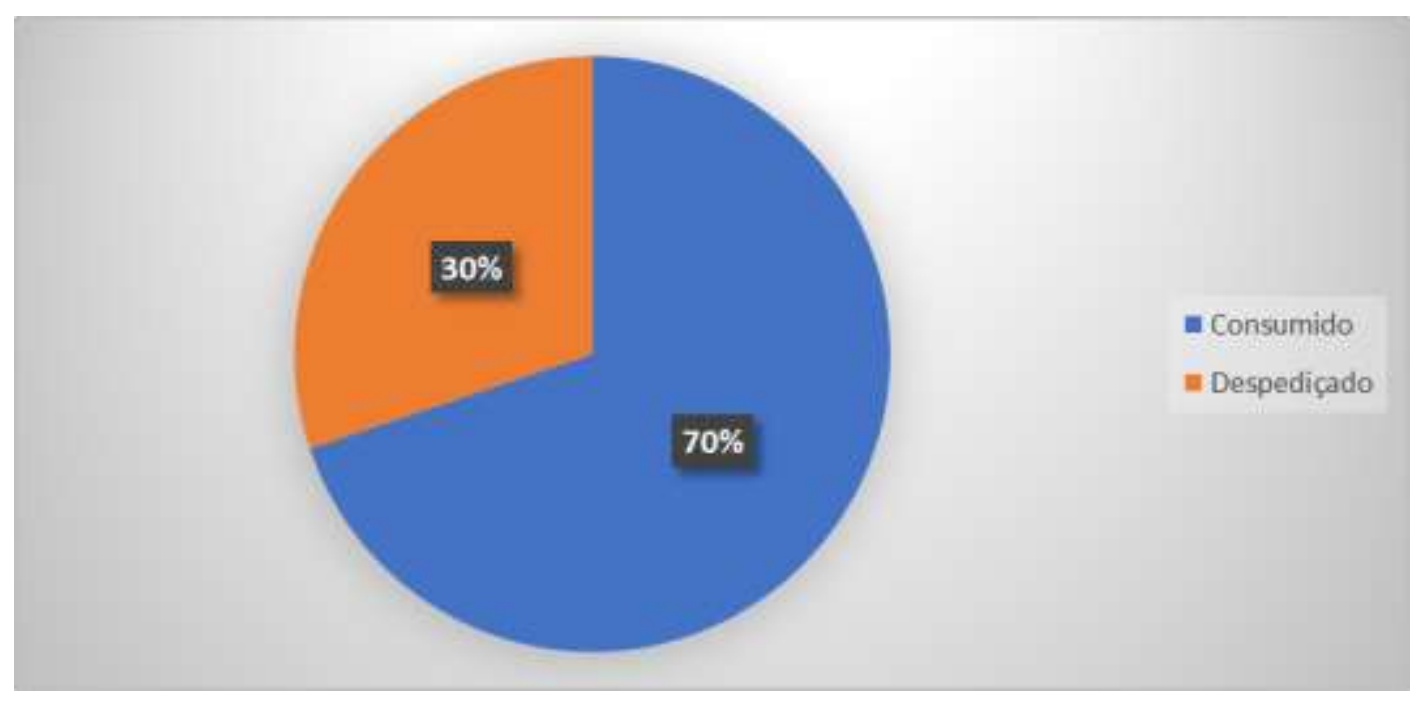

Fonte: Autores (2020).

Observa-se que foram distribuídos $47121 \mathrm{~g}$ de alimentos $(100 \%)$ e destes apenas $70 \%$ ( $\mathrm{n}=32922 \mathrm{~g}$ ) foram consumidos gerando, desse modo, um desperdício de 30\% ( $\mathrm{n}=14199 \mathrm{~g})$ que foram depositados nas lixeiras do hospital, ocasionando perdas econômicas e impactos ambientais.

Na literatura são admitidos como percentual de resto-ingestão (relação entre o resto devolvido nas bandejas e o consumido pelo comensal), taxas inferiores a 10\%. No entanto, quando o resultado se apresenta acima de $10 \%$ em coletividades sadias e $20 \%$ em enfermas, pressupõe-se que os cardápios estão inadequados, por serem mal planejados ou mal executados (Castro,2003). Segundo Anjos, Tavares, Bulhões e Mori (2017) a perda por resto-ingesta deve ser sempre avaliada, pois demonstra o compromisso do comensal relativamente ao valor do alimento servido e por ele desprezado.

A figura 7 apresenta o desperdício dos alimentos por todos os pacientes da pesquisa, comparando os valores de referência aceitável que são até $20 \%$ de desperdício para pacientes. 
Figura 7. Desperdício de alimentos pelos pacientes participantes da pesquisa.

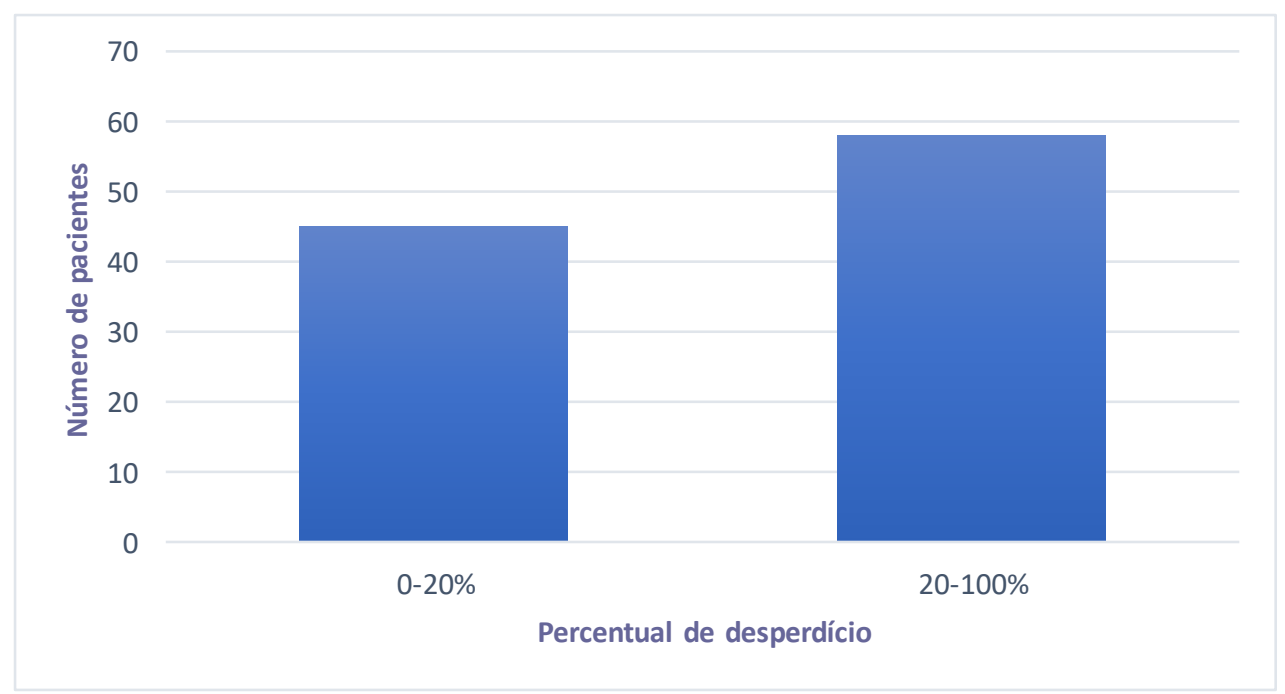

Fonte: Autores (2020).

Observa-se que 43,66\% ( $\mathrm{n}=45)$ dos pacientes desperdiçaram 0-20\% dos alimentos servidos; 56,31\%(n=58) desperdiçaram 20-100\% da refeição. Ou seja, a maioria dos pacientes apresentou um desperdício acima da média recomendada pela literatura que é de $20 \%$ para coletividade enferma.

O presente estudo corrobora com o que foi realizado nas enfermarias de um hospital de terapia intensiva na cidade de Portugal com intuito de verificar o desperdício nos pratos individuais dos pacientes. Em média, cada paciente joga fora $953 \mathrm{~g}$ de comida por dia, o que representa $35 \%$ da comida servida. Isto equivale a 8,7 mil toneladas de resíduos alimentares a serem eliminados todos os anos em hospitais de Portugal. Estas toneladas de alimentos transformados em resíduos representam perdas econômicas e impactos ambientais, estimando-se que 16,4 mil toneladas de CO2 (equivalente) e 35,3 milhões de euros sejam os indicadores nacionais anuais em Portugal. Isto significa que 0,5\% do orçamento nacional de saúde português é desperdiçado na forma de lixo alimentar (Dias-Ferreira; Santos; Oliveira, 2015).

Figura 8. Desperdício dos alimentos diferenciado pelos gêneros.

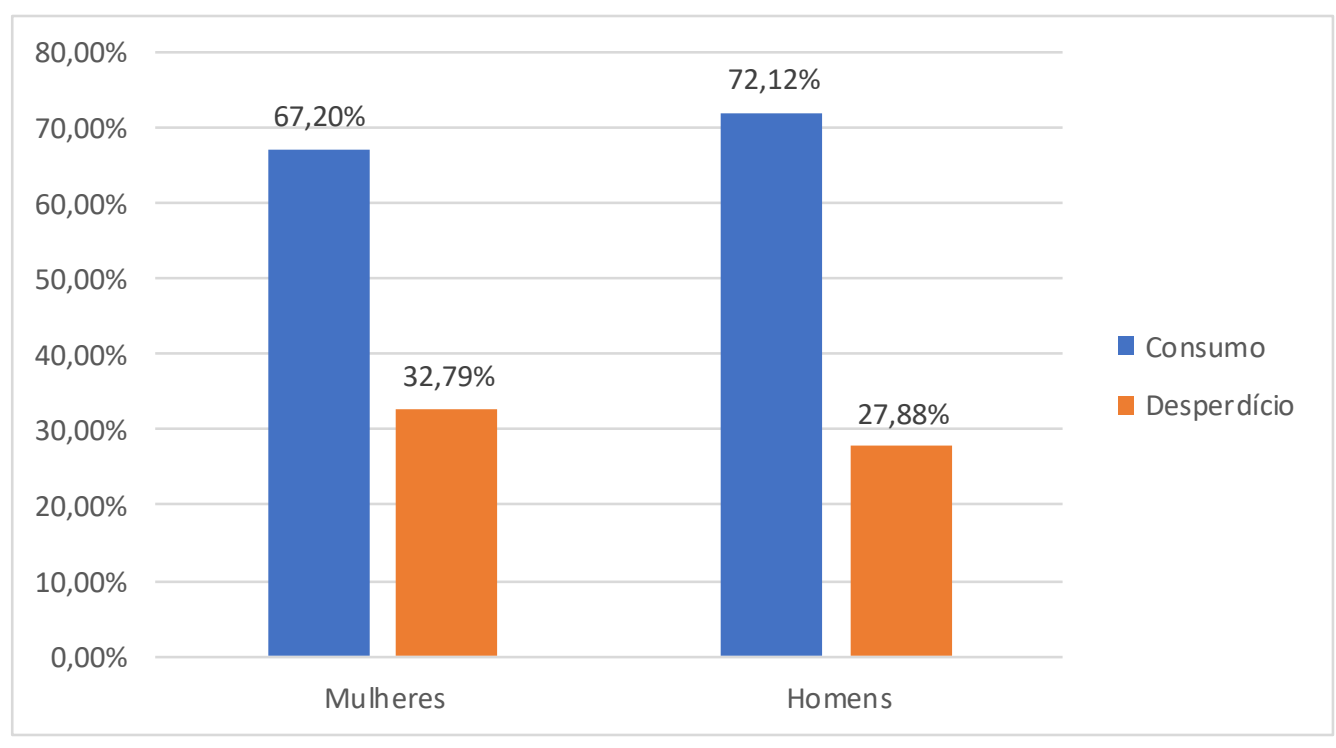

Fonte: Autores (2020). 
Quando comparado os desperdícios de alimentos realizados pelos dois gêneros (Figura 8), constatamos que o gênero masculino apresentou um menor desperdício, perfazendo um valor de $27,88 \%(n=7112 \mathrm{~g})$ do valor que foi a este ofertado $25509 \mathrm{~g}(100 \%)$. E o gênero feminino apresentou um maior desperdício com um valor de 32,79\% (n=7087g) em relação a $21612 \mathrm{~g}(100 \%)$ a elas ofertado. E nos dois casos ultrapassa a média de $20 \%$ de desperdício aceita para coletividades enfermas.

Para alguns autores essa comparação entre os gêneros deve ser realizada, porque os requisitos de certos nutrientes diferem para homens e mulheres (Nichols, Porter, Hammond, \& Arjmandi (2002). E o presente estudo apresenta como mesmo resultado que os estudos de Schiavone, Pelullo e Attena (2019), que relatou que as mulheres desperdiçaram mais alimentos do que os homens durante a internação $(59,1 \%$ vs. $38,2 \%)$ e o de Gomes, Saraiva, Esteves e Gonçalves (2020) que também apresenta maior desperdício entre as mulheres.

Os resultados encontrados na pesquisa mostraram que as refeições foram bem aceitas pelos pacientes internos do hospital, apesar das dietas hospitalares mais servidas geralmente não possuírem boa aceitação, no entanto, ainda se encontram graus de desperdício acima da média aceita pela literatura.

\section{Conclusão}

A análise em conjunto dos dados encontrados no presente estudo permite sugerir que os pacientes apresentaram boa aceitação da dieta oferecida, tanto em relação as variáveis analisadas quanto em relação a quantidade ingerida. Em contrapartida foi encontrado um grau de desperdício dos alimentos de $30 \%$ o que ultrapassa o limite aceitável de $20 \%$ para coletividade enferma. E como forma de evitar essas perdas será necessário um acompanhamento contínuo e ações educativas dentro do estabelecimento com intuito de amenizar esses desperdícios alimentares e evitar desnutrição nos pacientes, custos hospitalares e impactos ao ambiente.

Foi sugerido a equipe do hospital um plano de ação que minimize esse desperdício: realizar trimestralmente uma pesquisa de satisfação com os pacientes; colocar em murais a quantidade de desperdício diária dos alimentos, tirar e expor fotos de preparações para ajudarem nas prescrições médicas e doar os alimentos excedentes a pessoas com risco alimentar e nutricional, apoiado na Lei 14016 de 23 de junho de 2020.

\section{Referências}

Abreu, E. S. (2016). Gestão de unidades de alimentação e nutrição: um modo de fazer. São Paulo: Metha.

Anjos, ACS., Tavares, H. C., Bulhões, C. D. B. B., \& Mori, E (2017). Avaliação do índice de resto-ingestão em uma unidade de alimentação e nutrição. Revista E-Ciência,5(2),116-120.

Carino, S., Porter, J., Malekpour, S., \& Collins, J (2020). Environmental Sustainability of Hospital Foodservices across the Food Supply Chain: a systematic review. Journal of The Academy of Nutrition and Dietetics, 120(5),825-873.

Castro, M. D. A. S. (2003). Resto-ingesta e aceitação de refeições em uma unidade de alimentação e nutrição. Rev. Hig. Alim., 17(1).114-115.

Coloço, R. B, Holanda L. B, \& Portero-Mclellan K. C. (2009). Determinantes do grau de satisfação de pacientes internados referente a refeições oferecidas em um hospital universitário. Rev. Ciênc. Méd., 18(3),121-130.

Dias-Ferreira, C., Santos, T., \& Oliveira, V. (2015) Hospital food waste and environmental and economic indicators - A Portuguese case study. Waste Management, $46,146-154$.

Duarte, A., Marques, A. R., \& Colpo. E. (2016). Risco nutricional em pacientes hospitalizados durante o período de internação. Nutr. clín. diet. Hosp.,36(3), 146-152.

Folio, D., O'sullivan-Maillet, J., \& Touger-Decker, R. (2002). The Spoken Menu Concept of Patient Foodservice Delivery Systems Increases Overall Patient Satisfaction, Therapeutic and Tray Accuracy, and is Cost Neutral for Food and Labor. Journal of the American Dietetic Association,102(4),546-548.

Gomes, A., Saraiva, C., Esteves, A., \& Gonçalves, C.Evaluation of Hospital Food Waste-A Case Study in Portugal. Sustainability, $12(15)$. 6157-6157.

Macedo, J. L., Pereira, I.C., Oliveira, A. S. S. S., \& Magalhães, M. J. S. (2017). Adesão ao consumo da dieta hipossódica por indivíduos hipertensos hospitalizados. Reon Facema. 3(2):531-536. 
Research, Society and Development, v. 10, n. 1, e37410111913, 2021 (CC BY 4.0) | ISSN 2525-3409 | DOI: http://dx.doi.org/10.33448/rsd-v10i1.11913

Mancopes, R.,Gonçalves, B. F. T., Costa, C. C., Flores, T.G.,Santos, L. D. , \& Drozdz, D. R. C. (2013).Relato de caso: a importância da atuação multiprofissional na laringectomia supracricóide. Revista Cefac,15(5) 1379-1385.

Mosqueira, C.; Harris, L.R. (1996) Spoken Menu: a menu process catered to the patients food choices and present appetite. Journal of the American Dietetic Association, 96(9). 14-14.

Nascimento, T., Moreira, D. F., Carvalho, R. C. R., Pereira, M. A. O., Pereira, E. A. O., \& Vilela, B. S. (2017). Aceitabilidade das dietas orais de um hospital do Sul de Minas. Revista UIIPS-Unidade de Investigação do Instituto Politécnico de Santarém,5(5),141-148

Nichols, P. J., Porter, C.; Hammond, L., Arjmandi, B. H. (2002) Food Intake may be Determined by Plate Waste in a Retirement Living Center. Journal of the American Dietetic Association, 102(8), 1142-1144.

Pereira A.S. et al. (2018). Metodologia da pesquisa científica. [e-book]. Santa Maria. Ed. UAB/NTE/UFSM.https://repositorio.ufsm.br/bitstream/handle/1/15824/Lic_Computacao_Metodologia-Pesquisa-Cientifica.pdf?sequence=1.

Schiavone, S., Pelullo.C. P, \& Attena F., (2019). Patient Evaluation of Food Waste in Three Hospitals in Southern Italy. International Journal of Environmental Research and Public Health,16(22), 4330-4330.

Schiffman, S. S. (2009) Effects of Aging on the Human Taste System. Annals of The New York Academy of Sciences,1170(1), 725-729.

Silva, J.L., Marques A. P. M,. Leal, M. C. C., Alencar D. L., Melo, E. M. A. (2015) Fatores associados à desnutrição em idosos institucionalizados. Rev. Bras. Geriat. Gerotol.,18(2), 443-451.

Soares, J. C. (2015) Fatores associados à satisfação de dietas hospitalares: uma revisão bibliográfica. Monografia (Doutorado) - Curso de Especialização em Gestão e Organização Pública em Saúde, Universidade Federal de Santa Maria, RS. Brasil

Strasburg, V J., \& Jahno, V. D. (2020) Paradigmas das práticas de gestão ambiental no segmento de produção de refeições no Brasil. Eng. Sanit. Ambient., 22(1),3-12.

Vaillant, M.-F., Alligier, M., Baclet, N., Capelle, J., Dousseaux, M., Eyraud, E., Fayemendy, P., Flori, N., Guex, E., \& Hennequin, V. (2019).Recommandations sur les alimentations standard et thérapeutiques chez l'adulte en établissements de santé. Nutrition Clinique Et Métabolisme, 33(4), 235-253.

Vilchez-Cornejo, J., Romani, L., Reategui, S., Gomez-Rojas, E., \& Silva, C. (2020). Factores asociados a la realización de actividades de autocuidado en pacientes diabéticos en tres hospitales de Ucayali. Revista de La Facultad de Medicina Humana, 20 (2) 82-88.

Wendisch, C. (2010). Avaliação da Qualidade de Unidades de Alimentação e Nutrição (UAN) Hospitalares: construção de um instrumento. Dissertação de mestrado, Escola Nacional de Saúde Pública Sergio Arouca, Rio de Janeiro, Brasil

Williams, P., Walton, K. (2011) Plate waste in hospitals and strategies for change. e-SPEN, the European e-journal of clinical nutrition and metabolism, 6(6), $235-241$.

Wolff, L. S. Flynn, A., Xuan, Z., Errichetti, K. S., Walker, S.T.; Brodesky, \& M. K. (2020) The Effect of Integrating Primary Care And Mental Health Services On Diabetes And Depression. Medical Care, 59 (1),67-76. 